X-ray Conversion Efficiency of high-Z hohlraum wall materials for indirect drive ignition

E. Dewald, M. Rosen, S. H. Glenzer, L. J. Suter, F. Girard, J. P. Jadaud, J. Schein, C. G. Constantin, P. Neumayer, O. Landen

March 18, 2008

Physics of Plasma 
This document was prepared as an account of work sponsored by an agency of the United States government. Neither the United States government nor Lawrence Livermore National Security, LLC, nor any of their employees makes any warranty, expressed or implied, or assumes any legal liability or responsibility for the accuracy, completeness, or usefulness of any information, apparatus, product, or process disclosed, or represents that its use would not infringe privately owned rights. Reference herein to any specific commercial product, process, or service by trade name, trademark, manufacturer, or otherwise does not necessarily constitute or imply its endorsement, recommendation, or favoring by the United States government or Lawrence Livermore National Security, LLC. The views and opinions of authors expressed herein do not necessarily state or reflect those of the United States government or Lawrence Livermore National Security, LLC, and shall not be used for advertising or product endorsement purposes. 


\title{
X-ray Conversion Efficiency of high-Z hohlraum wall materials for \\ indirect drive ignition*
}

\author{
E.L. Dewald ${ }^{1}$, M. Rosen ${ }^{1}$, S.H. Glenzer ${ }^{1}$, L.J. Suter ${ }^{1}$, F. Girard ${ }^{2}$, J.P. Jadaud ${ }^{2}$, J. Schein ${ }^{3}$, \\ C. Constantin ${ }^{4}$, P. Neumayer ${ }^{1}$ and O.L. Landen ${ }^{1}$, \\ ${ }^{1}$ Lawrence Livermore National Laboratory, P.O. Box 808 Livermore, CA, 94551, USA \\ ${ }^{2}$ Commissariat à l'Énergie Atomique, Boîte Postale 12, 91680 Bruyères-le-Châtel, \\ France \\ ${ }^{3}$ University of German Armed Forces Munich, Werner-Heisenberg Dr., D-85577 \\ Neubiberg, Germany \\ ${ }^{4}$ Physics Department, University of California Los Angeles,Box 951547, Los Angeles, \\ CA, 90095, USA
}

\section{Abstract}

We measure the conversion efficiency of $351 \mathrm{~nm}$ laser light to soft $\mathrm{x}$-rays $(0.1-5 \mathrm{keV})$ for $\mathrm{Au}, \mathrm{U}$ and high $\mathrm{Z}$ mixtures "cocktails" used for hohlraum wall materials in indirect drive ICF. We use spherical targets in a direct drive geometry, flattop laser pulses and laser smoothing with phase plates to achieve constant and uniform laser intensities of $10^{14}$ and $10^{15} \mathrm{~W} / \mathrm{cm}^{2}$ over the target surface that are relevant for the future ignition experiments on NIF. The absolute time and spectrally-resolved radiation flux is measured with a multichannel soft x-ray power diagnostic. The conversion efficiency is then calculated by dividing the measured x-ray power by the incident laser power from which the measured laser backscattering losses is subtracted. After $\sim 0.5 \mathrm{~ns}$, the time resolved $\mathrm{x}$-ray conversion efficiency reaches a slowly increasing plateau of $95 \%$ at $10^{14} \mathrm{~W} / \mathrm{cm}^{2}$ laser 
intensity and of $80 \%$ at $10^{15} \mathrm{~W} / \mathrm{cm}^{2}$. The M-band flux $(2-5 \mathrm{keV})$ is negligible at $10^{14}$ $\mathrm{W} / \mathrm{cm}^{2}$ reaching $\sim 1 \%$ of the total $\mathrm{x}$-ray flux for all target materials. In contrast, the Mband flux is significant and depends on the target material at $10^{15} \mathrm{~W} / \mathrm{cm}^{2}$ laser intensity, reaching values between $10 \%$ of the total flux for $\mathrm{U}$ and $27 \%$ for Au. Our LASNEX simulations show good agreement in conversion efficiency and radiated spectra with data when using XSN atomic physics model and a flux limiter of 0.15 , but they underestimate the generated M-band flux.

*This work was performed under the auspices of the U.S. Department of Energy by Lawrence Livermore National Laboratory under Contract DE-AC52-07NA27344.

PACS numbers: 52.50.Jm, 52.38.Ph,Dx, 52.70.Kz,La 


\section{Introduction}

In indirect drive inertial confinement fusion (ICF) experiments, intense laser or charged particle beams heat the interior of high-Z cylindrical cavities called hohlraums to efficiently generate soft x-rays. The role of the soft x-rays is to uniformly produce an ablation drive that compresses the DT filled capsule placed inside the hohlraum, driving it to ignition and burn [1]. Due to the relaxed requirements on laser-beam uniformity and reduced sensitivity to hydrodynamic instabilities the indirect drive scenario is the main approach for future ignition experiments at the National Ignition Facility (NIF) $[2,3,4]$. In such a hohlraum containing a capsule and heated by laser beams the power balance is given by $[5,6]$ :

$$
\eta_{\mathrm{CE}}\left(\mathrm{P}_{\text {laser }}-\mathrm{P}_{\text {backscatter }}\right)=\mathrm{P}_{\mathrm{walls}}+\mathrm{P}_{\mathrm{LEH}}+\mathrm{P}_{\text {capsule }}=\sigma \mathrm{T}_{\mathrm{R}}^{4}\left[(1-\alpha) \mathrm{A}_{\mathrm{wall}}+\mathrm{A}_{\mathrm{LEH}}+\mathrm{f}_{\text {capsule }} \mathrm{A}_{\text {capsule }}\right]
$$

where $\eta_{\mathrm{CE}}$ is the laser conversion efficiency into soft $\mathrm{x}$-rays $(<2 \mathrm{keV}), \mathrm{P}_{\text {laser }}$ is the incident laser power, $\mathrm{P}_{\text {backscatter }}$ is the backscattered light by parametric laser-plasma instabilities, $\mathrm{P}_{\text {capsule }}$ is the radiation absorbed by the capsule, $\mathrm{P}_{\mathrm{LEH}}$ is the radiation escaping through the hohlraum laser entrance holes (LEH) and $\mathrm{P}_{\text {walls }}$ is the radiation loss in the hohlraum walls. Furthermore, $\sigma$ is the Stefan-Boltzmann constant, $T_{R}$ is the hohlraum radiation temperature ( $\leq 300 \mathrm{eV}), \alpha$ is the x-ray albedo of the hohlraum wall [7], defined as the ratio between the re-emitted and incident soft x-ray flux at the hohlraum wall, $\mathrm{A}_{\text {wall }}$ and $\mathrm{A}_{\mathrm{LEH}}$ are the areas of the hohlraum wall and LEH's, $\mathrm{f}_{\text {capsule }}$ is the fraction of incident $\mathrm{x}$-ray flux that is absorbed by the capsule and $\mathrm{A}_{\text {capsule }}$ is the capsule area. 
For the NIF ICF designs [1], the hohlraum wall albedo and fraction of incident radiation absorbed by the capsule are time-dependent and reach values of about 0.9 at the peak of the x-ray drive. According to complex radiation hydrodynamic simulations [8], the required radiation absorbed by the capsule and the ratio between the capsule and wall areas are determined by other factors such as required capsule heating and radiation uniformity onto the capsule for a successful ignition experiment [1]. As shown by Eq.(1), for a constant radiation temperature $T_{R}$, the incident required laser power can be decreased by increasing the conversion efficiency $\eta_{\mathrm{CE}}$, decreasing the backscattered laser power by reducing laser-plasma interaction processes inside the hohlraums, and reducing radiation wall losses by choosing wall materials with high x-ray albedo $\alpha$.

Advanced hohlraum designs use high $\mathrm{Z}$ materials such as $\mathrm{Au}, \mathrm{U}$ or high- $\mathrm{Z}$ mixtures called cocktails [7] that have increased wall albedo and thus lower radiation losses. In a cocktail, the mix has an increased opacity and decreased heat capacity compared to its individual constituents and thus reduced radiation losses into the walls $[9,10]$. A higher wall albedo not only reduces required laser power, but also improves radiation uniformity at the capsule [1].

For these ICF hohlraum wall materials an accurate assessment of the laser conversion efficiency into x-rays and emission spectra is important for a good understanding of the hohlraum energetics [Eq.(1)] as well as of the radiation uniformity at the capsule for successful indirect drive ICF designs. This can be achieved through precise x-ray conversion efficiency measurements in open geometries which are not influenced by the x-ray albedo of the hohlraum walls. 
Experiments using planar foils are complex to interpret due to laser intensity variations onto the target, leading to transverse density and temperature gradients in the emitting laser plasmas, and thus to variable x-ray conversion efficiencies. In such experiments it is therefore quite difficult to assess the validity of the x-ray emission models used in our ICF designs. For these reasons, in the present work, the soft x-ray conversion efficiency was measured in a convergent direct drive-like set-up [11], on spheres made of $\mathrm{Au}$, cocktail and $U$ uniformly heated by laser beams at the Omega Laser Facility [12].

\section{Experimental set-up}

Figure 1 shows the experimental set-up for the soft x-ray conversion efficiency measurements. The spherical targets consist of $1 \mathrm{~mm}$ diameter plastic $(\mathrm{CH})$ spheres coated with 7 um thick $\mathrm{Au}$, cocktail (Au:U:Dy=0.2:0.6:0.2) or U. For each material, 60 drive laser beams at $351 \mathrm{~nm}$ wavelength uniformly distributed around the spheres heat the targets. Two different laser pulses were used with a total energy per beam and pulse length of $500 \mathrm{~J}$ for $1 \mathrm{~ns}$ and $180 \mathrm{~J}$ for $3 \mathrm{~ns}$, respectively, both with nominally a flat top pulse shape. Full aperture phase plates [13] producing a beam spot size of $800 \mu \mathrm{m}$ were used to ensure uniform target heating and low laser backscattering by parametric instabilities,. The number of heater beams and laser spot size relative to the target size give a high degree of beam overlapping on the target surface, i.e. each area of the target is heated by approximately 10 overlapped beams. In these conditions, the average laser intensity onto the target surface is $1.1 * 10^{15} \mathrm{~W} / \mathrm{cm}^{2}$ for the $1 \mathrm{~ns}$ pulse and $1.3^{*} 10^{14} \mathrm{~W} / \mathrm{cm}^{2}$ for 3 ns, respectively. Measurements with $U$ targets were performed only at $1.1 * 10^{15}$ 
$\mathrm{W} / \mathrm{cm}^{2}$ where significant differences between the $\mathrm{x}$-ray spectra of the three materials are expected.

The x-ray source uniformity is measured time and space resolved with a soft x-ray gated camera with 50 ps exposure, filtered with three different mirror-filter combinations to image separately the target emissivity for three photon energy bands centered at $450 \mathrm{eV}$ and $760 \mathrm{eV}$ and above $1.2 \mathrm{keV}$, as shown in Figure 2. For the $450 \mathrm{eV}$ band a combination of an $\mathrm{Al}$ mirror and a thin $\mathrm{V}$ filter is used. The $760 \mathrm{eV}$ energy band is measured with a thin $\mathrm{Zn}$ filter coupled to a Ge mirror and the emission of the targets at energies above 1.2 $\mathrm{keV}$ is measured with a $50 \mu \mathrm{m}$ Be filter.

The absolute soft $\mathrm{x}$-ray flux in the $100 \mathrm{eV}$ to $5 \mathrm{keV}$ spectral range was measured temporally resolved with the Dante broadband spectrometer [14]. Dante is a collection of absolutely calibrated vacuum x-ray diodes (XRD) filtered individually with edge filters and mirrors for different soft x-ray energy ranges up to $12 \mathrm{keV}$. The overall error in absolute flux measurement using Dante is estimated to be smaller than $\pm 7 \%$ and is attributed to the calibration of the x-ray diodes, mirrors and filters [15]. However, the relative error that is important for back to back comparison of the conversion efficiencies for the different materials is considerably smaller than the absolute error, $1-2 \%$ for radiation temperatures of $250 \mathrm{eV}$. The total flux measured by Dante is used to estimate the total $\mathrm{x}$-ray conversion efficiency from laser energy into x-rays, while the radiation flux $>2$ $\mathrm{keV}$ is used to measure the M-band flux for the materials mentioned above, i.e. the emission bands corresponding to the transitions from higher energy levels to $n=3$, where $n$ is the main quantum number. The M-band emission between 1.8 and $3.5 \mathrm{keV}$ is also measured time integrated with high spectral detail using a Henway spectrometer [16]. 
We measure the incident laser power and the light scattered off the spheres. The backscattered laser energy is measured with a full aperture backscatter system (FABS) [17] inside the final laser focusing lenses and by a near backscatter imager (NBI) outside the focusing lenses [18]. The scattered light measured with FABS and NBI is then subtracted from incident laser power to estimate the laser coupling onto the targets.

In addition to absolute $\mathrm{x}$-ray emission and laser backscattering measurements, plasma parameters were measured in the blow-off plasma using $4 \omega$ Thomson Scattering, but these results will be discussed elsewhere.

The laser coupling and x-ray flux are compared to LASNEX calculations [8] using different flux limiters and atomic physics models. The flux limiters and improved atomic physics models are then employed for better modeling of the hohlraum wall plasma in ICF designs.

\section{Experimental results}

Figure 3 shows gated images of the x-ray emission of the Au target measured for $1.1 * 10^{15}$ $\mathrm{W} / \mathrm{cm}^{2}$ laser intensity for the $450 \mathrm{eV}, 760 \mathrm{eV}$ and $>1.2 \mathrm{keV}$ energy bands. The images show uniform emission across the target surface due to constant laser intensity, as expected. Furthermore, while at $450 \mathrm{eV}$ and $760 \mathrm{eV}$ the $\mathrm{x}$-ray emission is uniform and constant across the image, it is limb brightened at $>1.2 \mathrm{keV}$. This can be explained by a reduced x-ray opacity of the laser plasma at higher photon energies. For all photon energies the spatial FWHM of the emission is constant and similar to the initial sphere diameter throughout the laser pulse duration. Cocktail and $U$ sphere emission shows a similar behavior to Au. 
The total and M-band $(<2 \mathrm{keV})$ soft $\mathrm{x}$-ray fluxes measured with Dante spectrometer obtained for both laser intensities are shown in Figure 4, for the various target materials. For a laser intensity of $1.3 * 10^{14} \mathrm{~W} / \mathrm{cm}^{2}$ (Fig. $\left.4 \mathrm{a}\right)$, the total radiation and M-band $(>2 \mathrm{keV})$ fluxes are similar for both Au and cocktails. The M-band flux is negligible, i.e. reaches peak values that are about 3\% of the total flux. Moreover, the M-band does not follow the temporal behavior of the total flux, peaking at the beginning of the $\mathrm{x}$-ray drive and then maintaining a constant value of $\sim 0.5 \%$ of the total flux at later times.

At $1.1 * 10^{15} \mathrm{~W} / \mathrm{cm}^{2}$ laser intensity (Figure $4 \mathrm{~b}$ ) the total soft $\mathrm{x}$-ray flux is also similar for all materials. This demonstrates that, at both laser intensities relevant for ICF, the primary xray emission of the laser plasma is similar for all the considered high- $Z$ hohlraum materials. Different from the low intensity case, the M-band $(2-5 \mathrm{keV})$ flux is significant and increases with the Au content in the target, ranging between $10 \%$ of the total flux for $\mathrm{U}$ target to $27 \%$ for $\mathrm{Au}$. Furthermore, the temporal behavior of the M-band flux follows that of the total flux.

We used the FABS and NBI laser scattering measurements to estimate the laser coupling in the target. The FABS calorimeters revealed that the scattered light from the target was mainly Brillouin scattering. Figure 5 shows a typical Brillouin time resolved spectrum measured for $1.1 * 10^{15} \mathrm{~W} / \mathrm{cm}^{2}$ laser intensity. For both laser intensities, the scattered light peaks at the beginning of the laser pulse and has a narrow spectrum with a $0.1 \AA$ blue shift at first that progressively moves towards the laser wavelength at later times (Fig. 5). The narrow spectrum suggests that the scattered light is mainly refracted laser light rather than Brillouin backscattering. 
For all target materials, the FABS calorimeters measured in the solid angle of the laser focusing lens $1.2 \%$ of the incident beam energy at $1.3 * 10^{14} \mathrm{~W} / \mathrm{cm}^{2}$ laser intensity and $2.5 \%$ at $1.1 * 10^{15} \mathrm{~W} / \mathrm{cm}^{2}$ intensity. Moreover, a second FABS with no heater beam measured similar scattered energy and spectrum as the one belonging to a heater beam. At the same time, the NBI images showed uniform scattered light distribution outside the beam focusing lenses.

The combined information from the two FABS and from NBI made us conclude that the light is scattered by the target uniformly in $4 \pi$. In consequence, to estimate the total laser energy scattered by the target, we multiply the values measured by FABS calorimeters with the solid angle ratio of the sphere and of the $f / 6.5$ of the laser cones, i.e. of FABS. The total scattered energy and the time history of the scattered light (Fig. 5) are used to estimate the total scattered power from the target. Implicitly, the laser power coupled in the target is the subtraction of the scattered power from the incident laser power, as shown in Figure 6 for both laser intensities.

Finally, the ratio between x-ray flux measured by Dante (Fig. 4) and the laser power coupled to the target (Fig. 6) gives us the time resolved x-ray conversion efficiency shown in Figure 7. At $1.3 * 10^{14} \mathrm{~W} / \mathrm{cm}^{2}$ laser intensity, after the first $0.5 \mathrm{~ns}$, the conversion efficiency reaches a slowly increasing value averaging $97 \%$ over the last $1 / 3$ of the laser drive. The fairly constant conversion efficiency is the result of balancing plasma heating by inverse bremsstrahlung absorption [19] with the radiative and hydrodynamic losses. The slight increase of the plateau is ascribed by the reduction in the diffusive radiative losses into the sphere bulk material. (i.e. increasing $\mathrm{x}$-ray albedo). Both cocktails and $\mathrm{Au}$ showed a similar behavior in the absorbed laser power history and consequently in the 
time resolved conversion efficiency. At $1.1 * 10^{15} \mathrm{~W} / \mathrm{cm}^{2}$ intensity we observed a similar temporal behavior of the conversion efficiency, (Fig. 7b) which reaches average values of about $78 \%$ over the last $1 / 3$ of the laser pulse for the various high- $Z$ materials. Furthermore, we obtained a good agreement of the time resolved conversion efficiency with 2D LASNEX simulations using XSN atomic physics models and a flux limiter of 0.15 as will be discussed in the following section.

\section{Data comparison with calculations and discussion}

We used the absorbed laser light and the x-ray production to benchmark the atomic physics models and electron heat transport models used in LASNEX calculations of the high-Z laser plasmas. In previous studies [20,21], we have tested a variety of detailed atomic physics models, including the simple average atom (XSN) model and, we have tested flux limiters in the 0.05-0.15 range. In this paper, for simplicity we use XSN model, and we study the dependence of calculated results on the value of the flux limiter. We observed that, while at $1.3 * 10^{14} \mathrm{~W} / \mathrm{cm}^{2}$ laser intensity the calculated results are relatively insensitive to flux limiters, at $1.1 * 10^{15} \mathrm{~W} / \mathrm{cm}^{2}$ the calculated results depend strongly on the choice of flux limiters as shown in Figure 8. The plots of absorbed laser light and total x-ray energy calculated with LASNEX show that the best agreement with the experimental data is obtained for a flux limiter near 0.15. For an XSN atomic physics model and a flux limiter of 0.15 we compare the calculated time history of the absorbed laser power and of the generated x-ray flux for ideal flattop laser pulses shown in Figure 9 with the experimental results (Figs 6 and 7). When comparing the experimental data with the calculations we notice that for both laser intensities onto the target, the calculated 
absorbed laser power, emitted laser flux and implicitly conversion efficiency agree with the experimental data to within measurement error bars.

Besides the total x-ray flux it is important to assess how well the simulations reproduce the spectral content of the generated $\mathrm{x}$-rays. Figures $10 \mathrm{a}$ and $\mathrm{b}$ show the $\mathrm{x}$-ray spectra measured by Dante broad band spectrometer for the two laser intensities and various laser materials at the peak of the x-ray flux (Fig. 3). We observe that, at both laser intensities the cocktails have the most Planckian spectra. Significant differences between the M-band content are observed between the studied materials at $1.1 * 10^{15} \mathrm{~W} / \mathrm{cm}^{2}$ laser intensity. We have generated synthetic Dante x-ray spectra from our LASNEX simulations using the XSN atomic physics model and flux limiter of 0.15 (Figure 9) shown in Figures $10 \mathrm{c}$ and $\mathrm{d}$ for the two ICF relevant laser intensities. Considering the broad spectral bandwidth of Dante, the shapes of the simulated spectra are in good agreement with the Dante data for both laser intensities. Moreover, the calculations reproduce the M-band flux differences between the high- $\mathrm{Z}$ materials at $1.1 * 10^{15} \mathrm{~W} / \mathrm{cm}^{2}$ laser intensity (Figs $10 \mathrm{~b}$ and d).

The Dante broadband spectra are the result of an unfolding process. Thus we have used the time integrated x-ray spectra measured with the Henway spectrometer, shown in Figure 11 which provides high spectral detail in the 1.8 to $3.5 \mathrm{keV}$ range, to crosscheck the accuracy of Dante spectra. The measured spectra for cocktails and Au show a local maximum at $2.6 \mathrm{keV}$ corresponding to $\mathrm{Au} 4-3 \mathrm{M}$-band transitions. The ratio between the time integrated Dante M-band fluxes for Au and cocktails at both laser intensities agrees with the ratio between spectral integrated Henway fluxes. Furthermore, the high resolution Henway spectra are consistent with the low resolution Dante spectra in the M-band range 
for the different high- $\mathrm{Z}$ materials especially for $1.1 * 10^{15} \mathrm{~W} / \mathrm{cm}^{2}$ laser intensity for which the radiation flux in the M-band range is significant.

\section{Summary}

We measured the time resolved conversion efficiencies from $3 \mathrm{w}$ laser light into $\mathrm{x}$-rays for the high-Z hohlraum wall materials under consideration for indirect drive ICF for $10^{14}$ and $10^{15} \mathrm{~W} / \mathrm{cm}^{2}$ laser intensities onto the target. In the present experiments uniform laser irradiation of the target was obtained by using phase plates for the drive laser beams. In these conditions the high- $Z$ plasmas have practically no transverse temperature and density gradients over the spherical target surface, yielding uniform soft x-ray emission over the entire $4 \pi$ sphere solid angle viewed. Thus the x-ray conversion efficiency of these spherically symmetric plasmas is not distorted by the colder plasma regions at different temperatures. However, gradients in plasma conditions are generated in the radial direction, resulting in variable limb brightening as shown in the x-ray images (Fig. 2). Besides laser uniformity onto target and time resolution, another significant difference to previous studies [11] performed in $\mathrm{Au}$ is the assessment of laser coupling onto the targets by measuring the backscattered light with FABS and NBI. Using the laser coupled into the target rather than the incident laser power yielded higher conversion efficiency than in [11]. Furthermore, the conversion efficiency reaches a fairly constant plateau after $0.5 \mathrm{~ns}$ from the beginning of the laser pulse, when the radiative and hydrodynamic losses in the high-Z plasmas are balanced by the laser plasma heating by inverse bremmsstrahlung [19]. The plateau is slightly increasing in time due to the increasing $\mathrm{x}$ ray albedo [10] that causes a reduction of radiative losses in the bulk target material. 
The measured and calculated total and M-band x-ray conversion efficiencies over the last $1 / 3$ of the pulse are summarized in Figure 12 . For $10^{15} \mathrm{~W} / \mathrm{cm}^{2}$ laser intensity, the measured conversion efficiencies are in the $78-80 \pm 7 \%$ range for $\mathrm{Au}, \mathrm{U}$ and cocktails. The M-band ( $>2 \mathrm{keV}$ ) conversion efficiency is $7-20 \%$ and increases with the Au content. For

$10^{14} \mathrm{~W} / \mathrm{cm}^{2}$ laser intensity, the measured conversion efficiencies are in the $95-97 \pm 7 \%$ range for $\mathrm{Au}$ and cocktails, with a negligible M-band conversion efficiency (1\%). LASNEX calculations using XSN atomic physics model and a flux limiter of 0.15 are in good agreement with total x-ray flux and laser coupling measurements within the measurement error bars. Furthermore, the measured spectra are reproduced fairly well by calculations which, however, underestimate the M-band flux at $10^{15}$ laser intensity, for all target materials. As a next step, the atomic physics models used to simulate the hohlraum wall laser plasmas in ICF designs on NIF [1] will be improved, which is important to assess the M-band preheat of the ICF capsules.

\section{References}

[1] J.D. Lindl, Phys. Plasmas 2, 3933 (1995); J.D. Lindl et al., Phys. Plasmas 11, 339 (2004).

[2] G.H. Miller, E.I. Moses and C.R. Wuest, Nucl. Fusion 44, 228 (2004).

[3] E.L. Dewald et al. Phys. Rev. Lett. 95, 215004 (2005).

[4] S.H. Glenzer et al. Nature Phys. 3, 716 (2007).

[5] L.J. Suter et al., Phys. Plasmas 3, 2057 (1996).

[6] S.H. Glenzer et al., Phys. Rev. Lett. 80, 2845 (1998).

[7] T.J. Orzechowski et al., Phys. Rev. Lett. 77, 3345 (1996). 
[8] G.B. Zimmerman and W.L. Kruer, Comm. Plasma Phys. Contr. Fusion 2, 51 (1975).

[9] J. Schein, O. Jones, M. Rosen et al., Phys. Rev. Lett. 98, 175003 (2007).

[10] O.S. Jones et al., Phys. Plasmas 14, 056311 (2007).

[11] P.D. Goldstone, S.R. Goldman, W.C. Mead, J.A. Cobble, et al., Phys. Rev. Lett. 59, 56 (1987).

[12] J. M. Soures, et al. Phys. Plasmas 3 (5), 2108 (1996).

[13] D. H. Munro, S. N. Dixit, A. B. Langdon, J. R. Murray, Appl. Opt. 43, 6639 (2004).

[14] E.L. Dewald et al., Rev. Sci. Instrum. 75, 3759 (2004).

[15] K.M. Campbell, F.A. Weber, E.L. Dewald, S.H. Glenzer, O.L. Landen, R.E. Turner, P.A. Waide, Rev. Sci. Instrum. 75, 3759 (2004).

[16] L.N. Koppel and J.D. Eckels, LLNL Report UCRL-79781 (1977).

[17] D. Froula et al., Rev. Sci. Instrum. 75, 4168 (2004).

[18] A.J. Mackinnon et al., Rev. Sci. Instrum. 75, 4183 (2004).

[19] T.W. Johnston and J.M. Dawson, Phys. Fluids 16, 722 (1973).

[20] D. E. Post, et al., At. Ata Nucl. Data Tables 20, 397 (1977).

[21] D. Colombant et al., Phys. Rev. E. 57, 3411 (1998). 


\section{Figure captions}

Fig. 1 Experimental set-up

Fig. 2 Filter-mirror transmissions for the gated soft x-ray imaging

Fig. 3 Gated soft $\mathrm{x}$-ray images of Au targets at $10^{15} \mathrm{~W} / \mathrm{cm}^{2}$ laser intensity at $\mathrm{t}=0.7 \mathrm{~ns}$

Fig. 4 Total and M-band ( $>2 \mathrm{keV}$ ) soft x-ray fluxes measured by the Dante

spectrometer for (a) $3 \mathrm{~ns}, 10^{14} \mathrm{~W} / \mathrm{cm}^{2}$ incident intensity and (b) for $1 \mathrm{~ns}, 10^{15} \mathrm{~W} / \mathrm{cm}^{2}$

incident intensity

Fig. 5 Typical light backscattering spectrum from the target for $1.1 \cdot 10^{15} \mathrm{~W} / \mathrm{cm}^{2}$ laser intensity

Fig. 6 Total incident laser power(black), scattered light(magenta) and the resulting laser coupling in the target(blue) at (a) $1.3 \cdot 10^{14} \mathrm{~W} / \mathrm{cm}^{2}$ laser intensity and (b) at $1.1 \cdot 10^{15}$

$\mathrm{W} / \mathrm{cm}^{2}$ intensities onto the targets

Fig. 7 Laser power coupled onto the target(blue), total x-ray flux measured by the Dante spectrometer(red) and the resulting time resolved x-ray conversion efficiency(green) for

(a) $1.3 \cdot 10^{14} \mathrm{~W} / \mathrm{cm}^{2}$ laser intensity onto the target (b) at $1.1 \cdot 10^{15} \mathrm{~W} / \mathrm{cm}^{2}$ intensity; the plot for $10^{15} \mathrm{~W} / \mathrm{cm}^{2}$ intensity includes time resolved conversion efficiency as calculated by LASNEX using XSN and a flux limiter of 0.15(black).

Fig. 8 Dependence of calculated laser absorption and x-ray production on the flux limiter for $\mathrm{Au}$ target at $10^{15} \mathrm{~W} / \mathrm{cm}^{2}$ intensity (LASNEX $1 \mathrm{D}$ calculations)

Fig. 9 Calculated absorbed laser power(blue), generated x-ray flux(red) and the resulting $\mathrm{X}$-ray conversion efficiency (green) for flattop incident laser power at (a) $1.3 \cdot 10^{14} \mathrm{~W} / \mathrm{cm}^{2}$ laser intensity onto the target and for (b) $1.1 \cdot 10^{15} \mathrm{~W} / \mathrm{cm}^{2}$ intensity ; incident laser power is also shown (black line) at $1.1 \cdot 10^{15} \mathrm{~W} / \mathrm{cm}^{2}$ intensity. 
Fig. 10 Measured and calculated x-ray spectra at the peak of the x-ray drive (Fig. 3) for (a,c) $1.3 \cdot 10^{14} \mathrm{~W} / \mathrm{cm}^{2}$ laser intensity onto target and $(\mathrm{b}, \mathrm{d})$ for $1.1 \cdot 10^{15} \mathrm{~W} / \mathrm{cm}^{2}$ intensity Fig. 11 X-ray spectra measured by Henway spectrometer for Au and cocktails for both laser intensities

Fig. 12 Total (squares) and M-band ( $>2 \mathrm{keV}$, circles) $\mathrm{x}$-ray conversion efficiencies averaged over the last $1 / 3$ of the laser pulse for the different high- $Z$ materials and laser intensities; full points show experimental data and empty points are LASNEX simulations using XSN model and a flux limiter of 0.15 . 
Figure 1

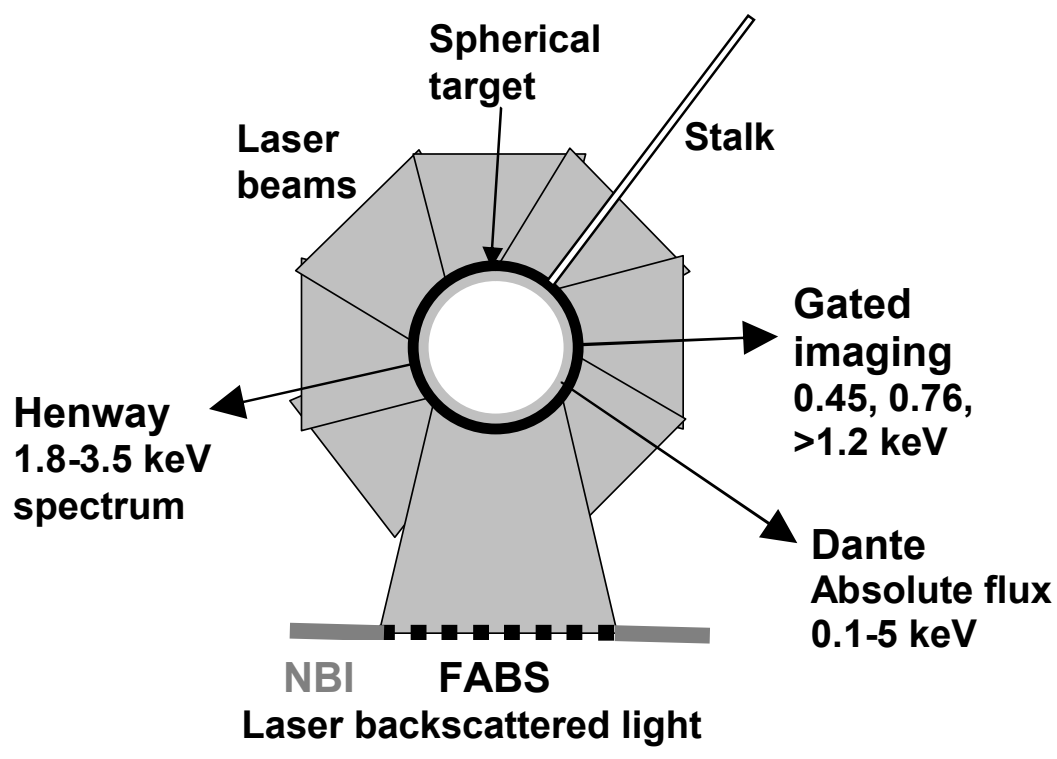


Figure 2

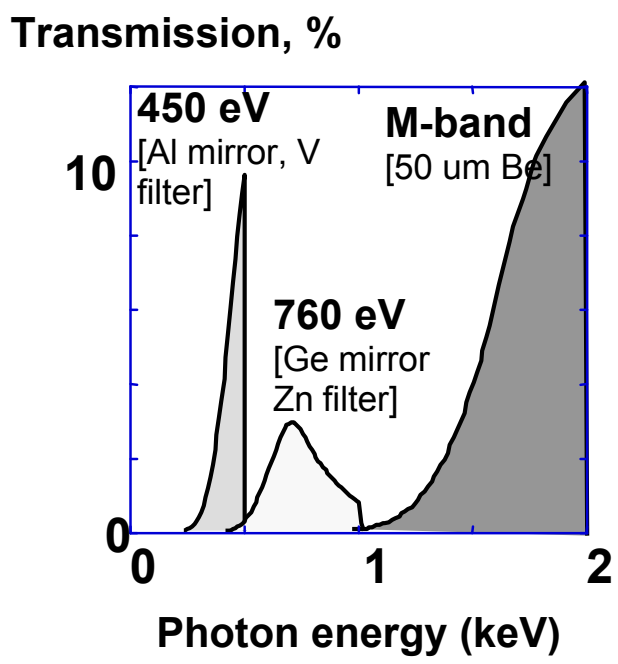


Figure 3
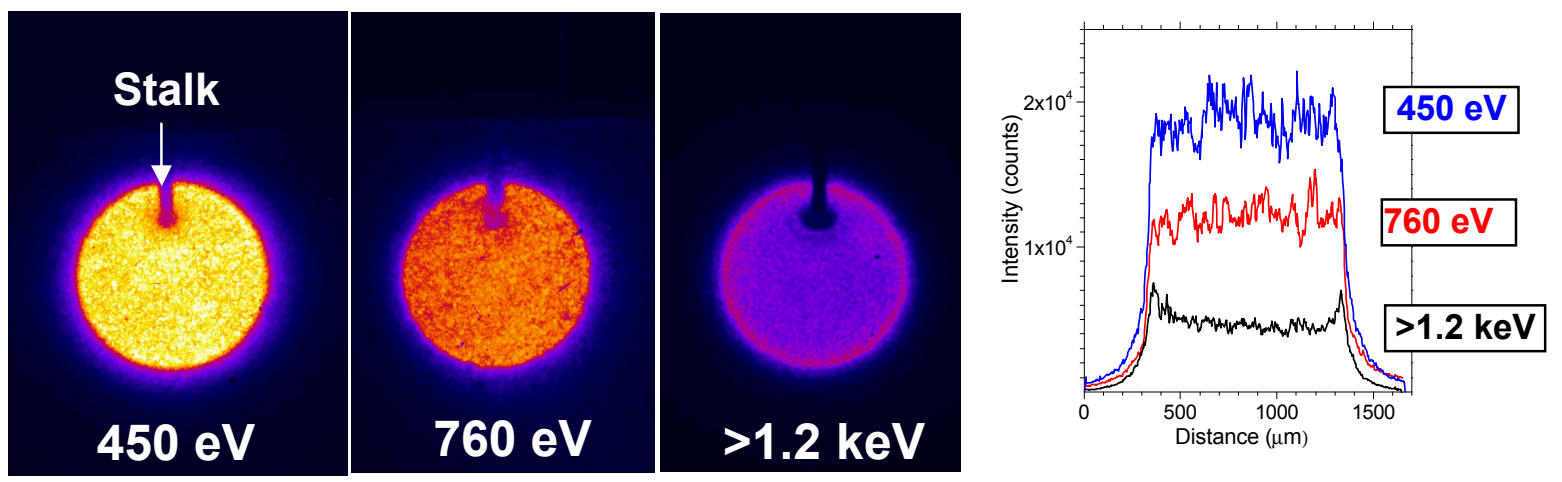
Figure 4

$a$

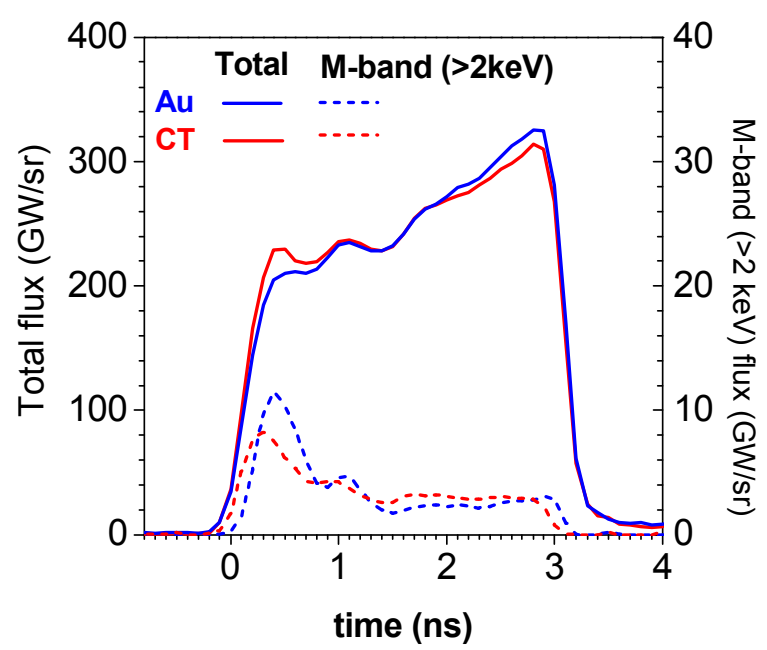

b

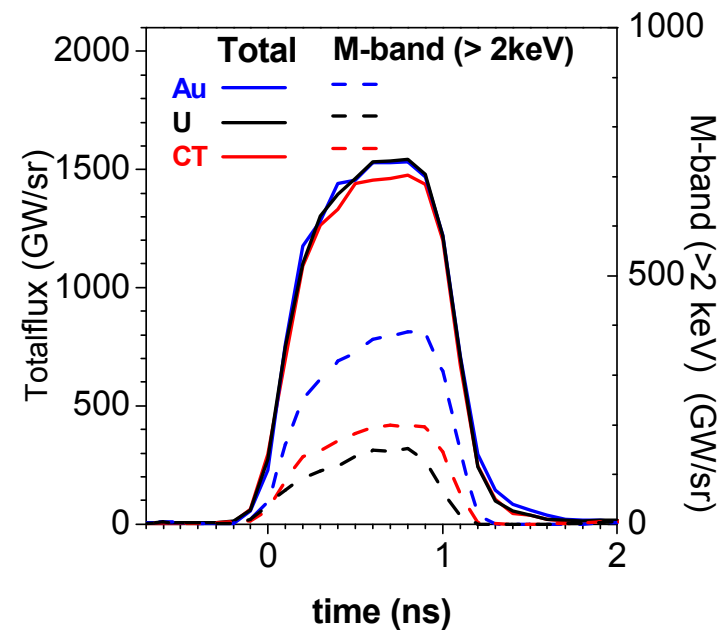


Figure 5

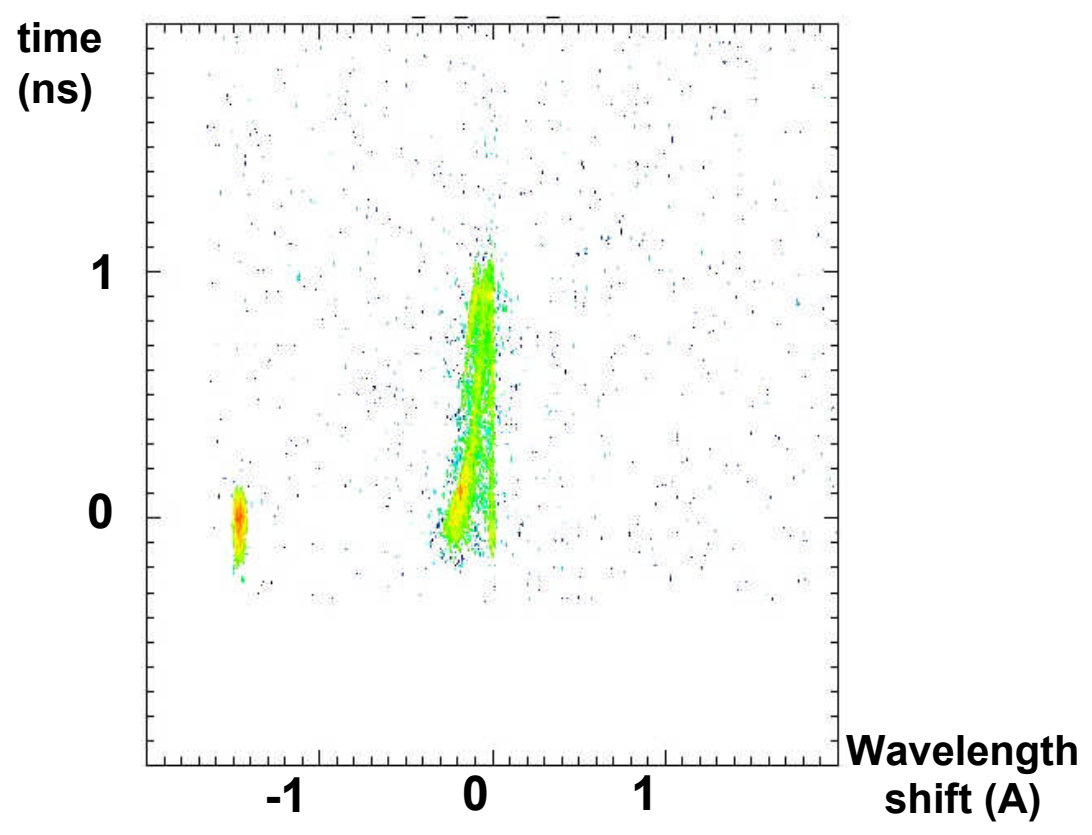


Figure 6

(a)

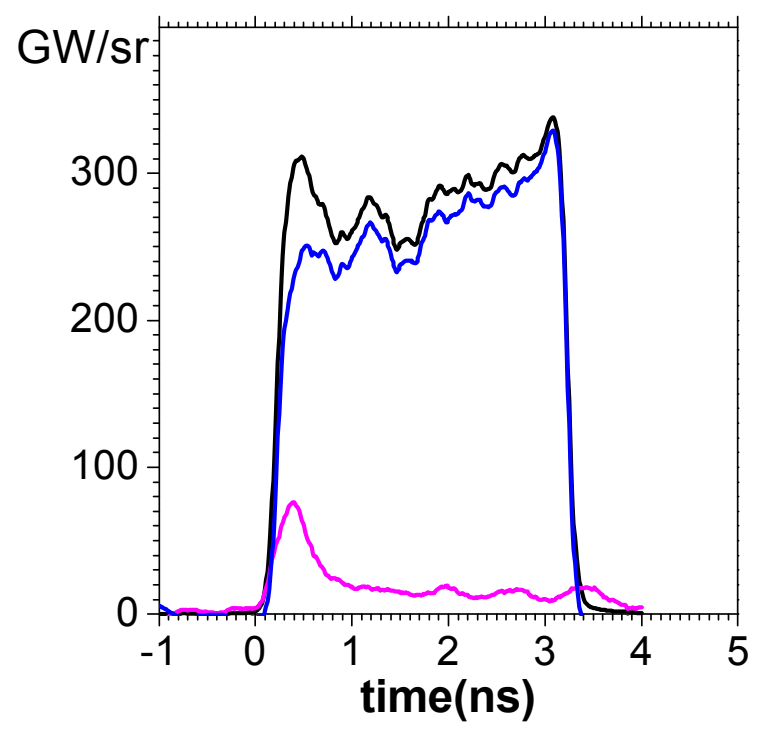

(b)

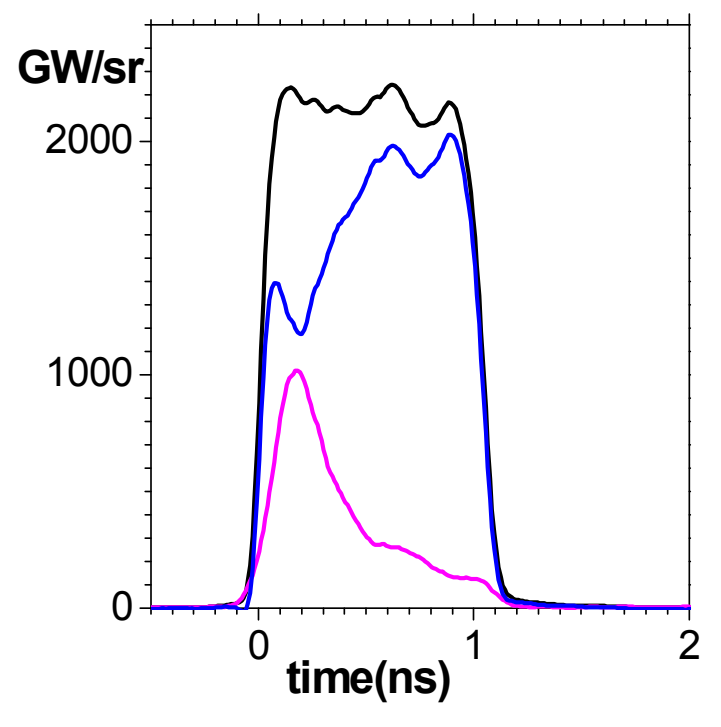


Figure 7

(a)

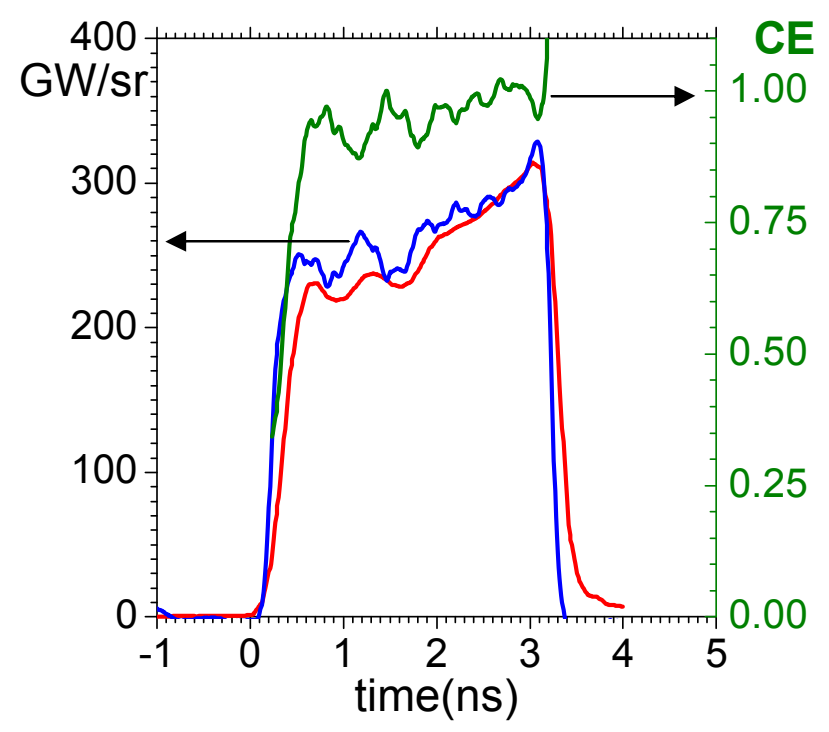

(b

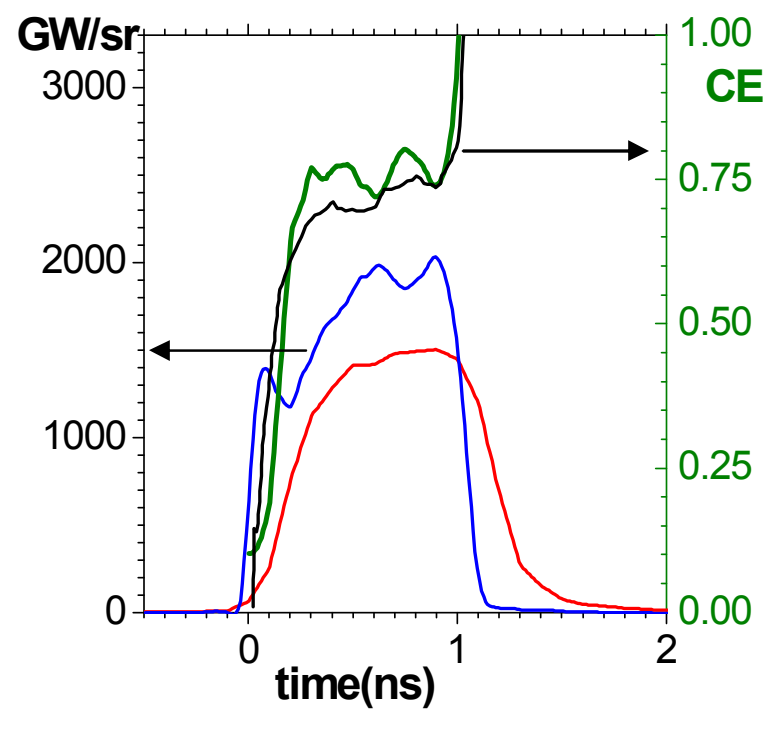




\section{Figure 8}

(a)

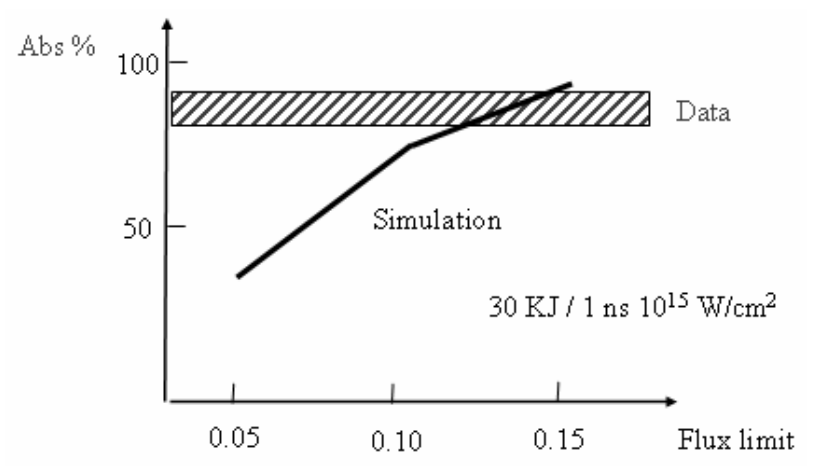

(b)

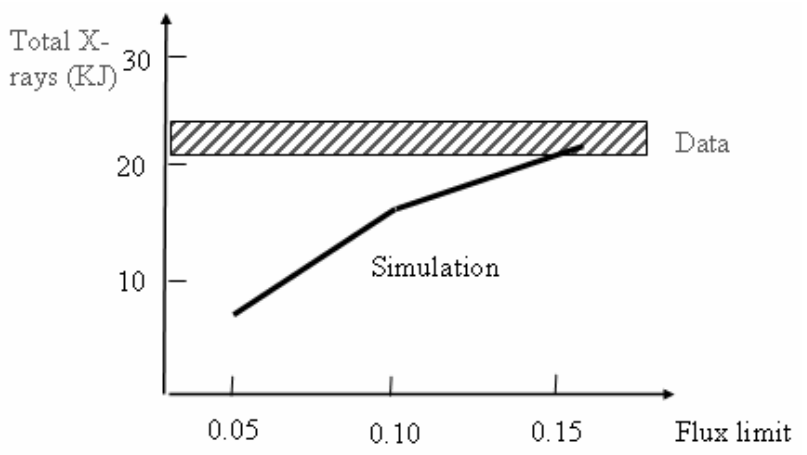


Figure 9

(a)

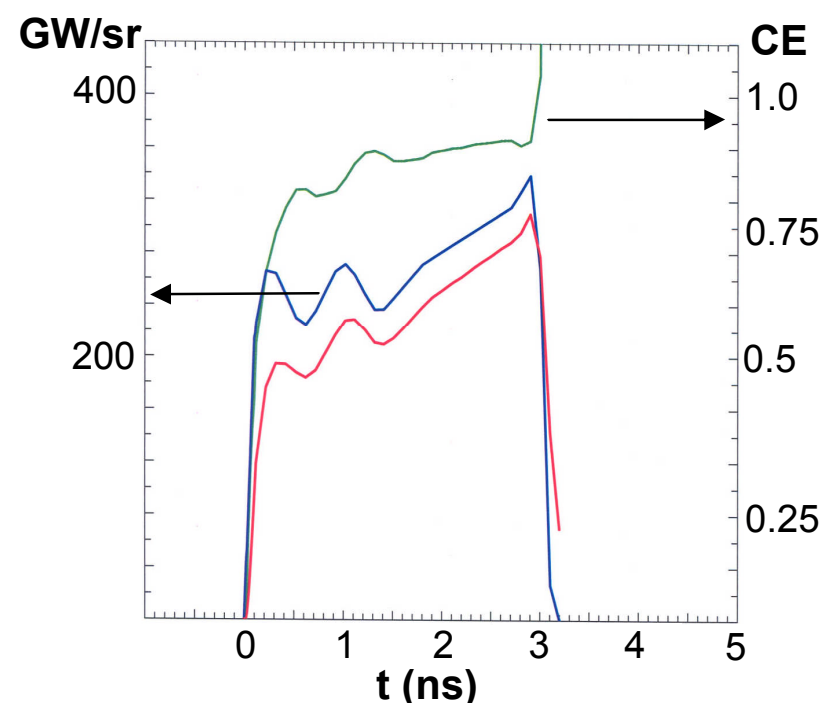

(b)

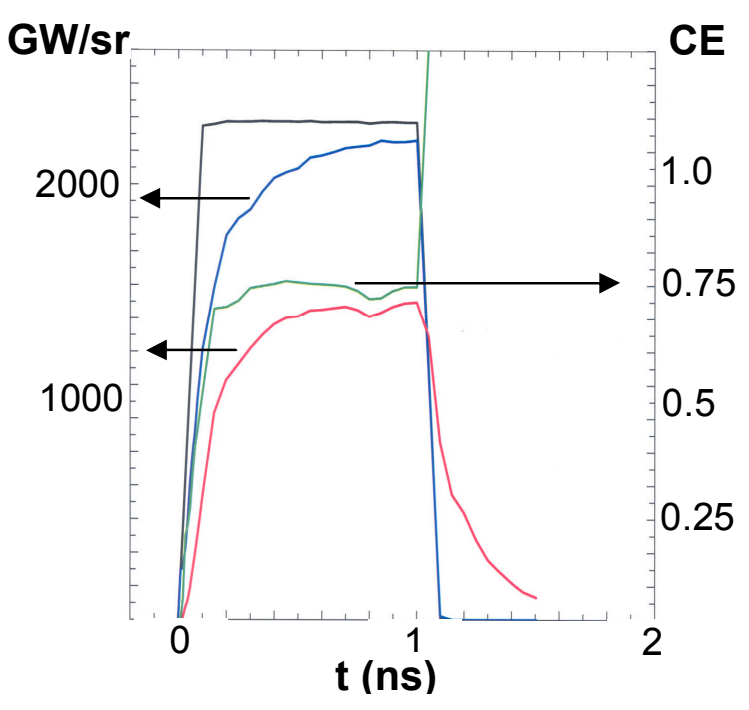


Figure 10
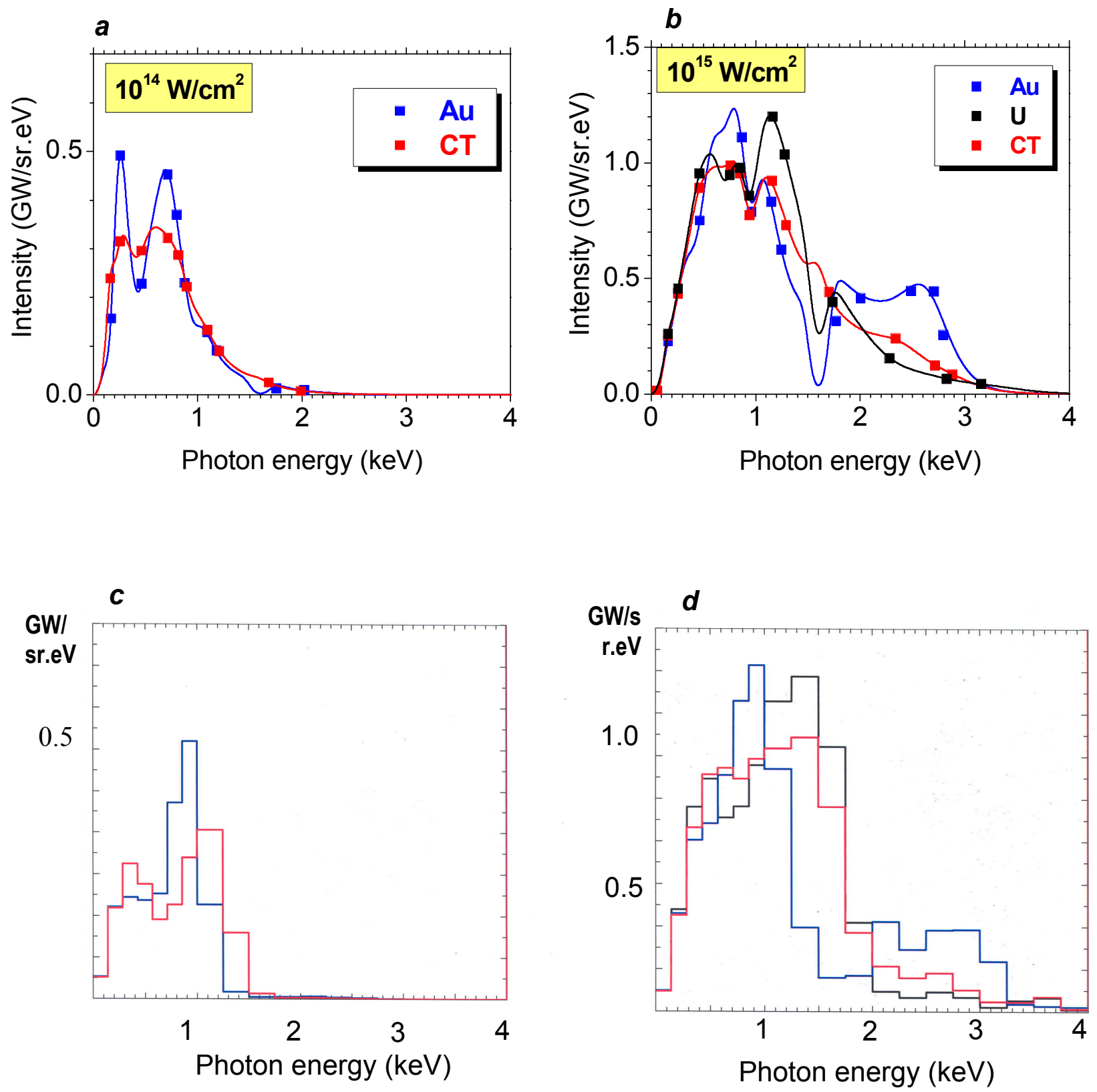
Figure 11
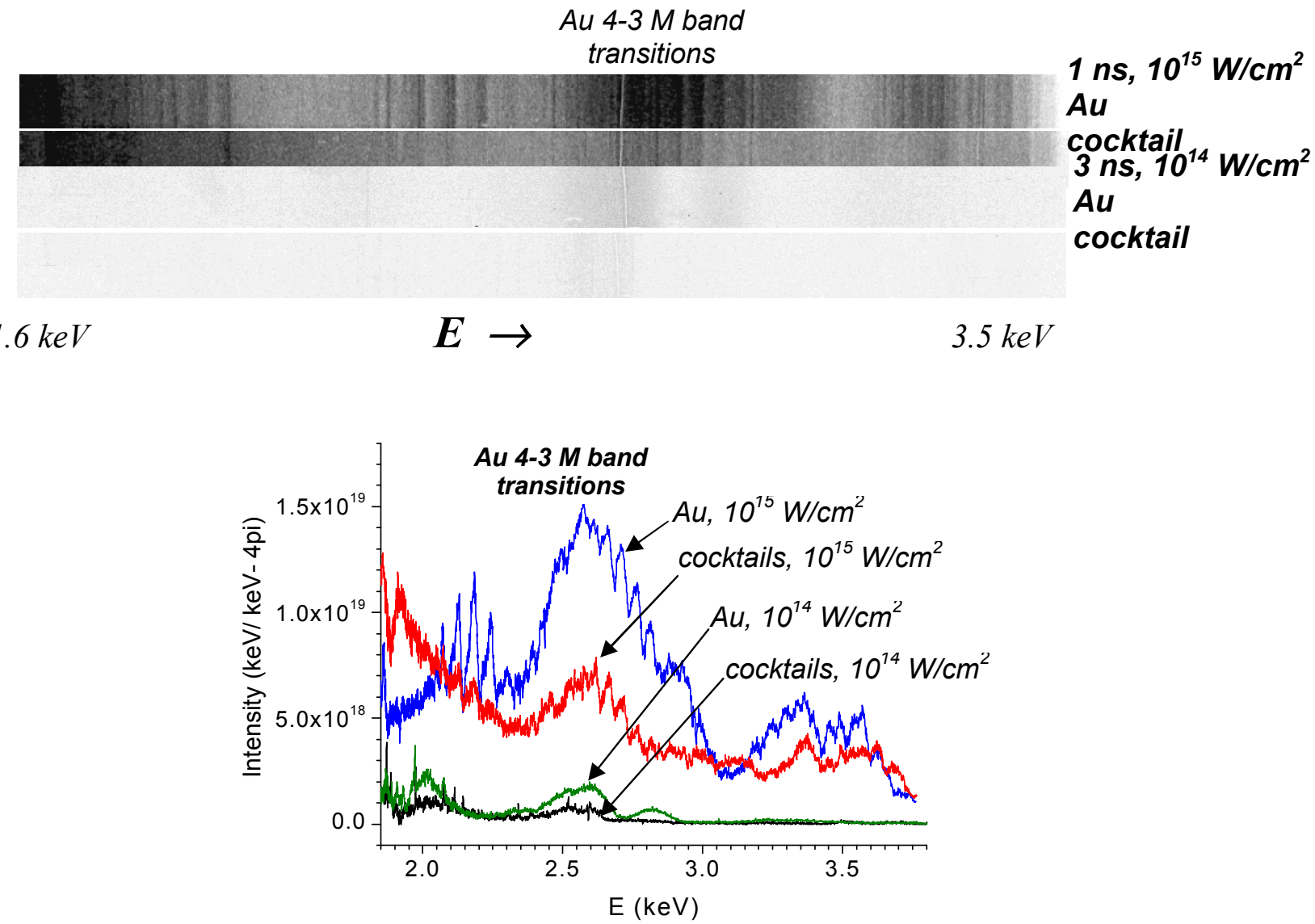
Figure 12

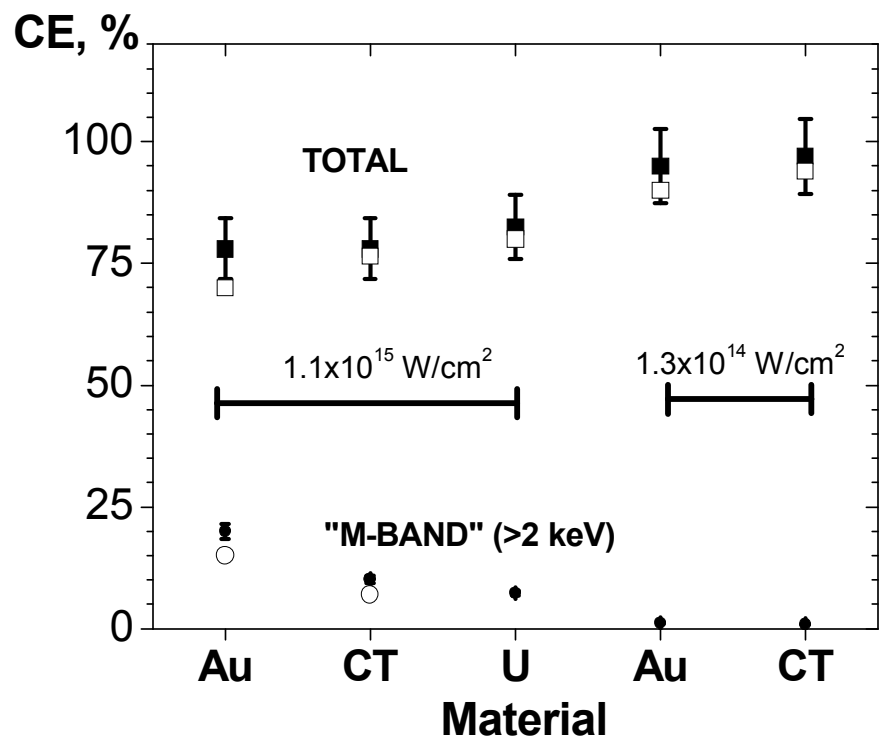

